\title{
Spin Relaxation and g-factor Manipulation in Quantum Dots
}

\author{
Carlos F. Destefani * and Sergio E. Ulloa \\ Department of Physics and Astronomy and Nanoscale and Quantum Phenomena Institute, Ohio University, Athens, Ohio 45701, USA
}

Received on 4 April, 2005

\begin{abstract}
Phonon-induced spin relaxation rates and electron g-factor tuning of quantum dots are studied as function of in-plane and perpendicular magnetic fields for different dot sizes. We consider Rashba and Dresselhaus spinorbit mixing in wide and narrow-gap semiconductors, and show how Zeeman sublevels can relax via piezoelectric (GaAs) and deformation ( $\mathrm{InSb}$ ) potential coupling to acoustic phonons. We find that strong confinement may induce minima in the rates at particular values of the magnetic field (due to a magnetic field-induced cancellation of the spin-orbit effects), where spin relaxation times can reach seconds. We also report on $g$-factor anisotropy. We obtain good agreement with available experimental values.
\end{abstract}

Keywords: g-factor; Phonon-induced spin relaxation; Quantum dots

Since the proposal of a qubit based on the electron spin of quantum dots (QDs) [1], much work has been done to understand the processes that may cause their relaxation, since long coherence times are required. One of those processes is related to the phonon-induced spin-flip rates of Zeeman sublevels in QDs in magnetic fields, where the spin purity of the levels is broken by the spin-orbit (SO) interaction. A recent experiment [2] has shown a spin relaxation time $\approx 0.55 \mathrm{~ms}$ at an in-plane field of $10 \mathrm{~T}$ in a GaAs QD defined in a 2DEG. In general, $\mathrm{SO}$ effects have been considered via perturbation theory [3], although exact treatments have also been presented $[4,5]$. The perturbative approach, which includes only a few states, has been called into question by the demonstration that a larger basis is needed in order to achieve convergence even for the lowest QD states when the QD vertical width is narrow [4], as a complex interplay between different energy scales can be present [6]. Insights on the purity of the spin degree of freedom of electrons in QDs can also be extracted from measurements of their effective $g$-factor, e.g., by means of capacitance [7] and energy [8] spectroscopies.

In this work we study spin-flip rates and $g$-factor tuning in QDs under SO influence. Our goal is to compare wide and narrow-gap materials under in-plane and perpendicular magnetic fields, at different QD sizes.

The QD is defined by an in-plane parabolic confinement, $V(\mathbf{r})=m \omega_{0}^{2} \mathbf{r}^{2} / 2$, where $m\left(\omega_{0}=E_{0} / \hbar\right)$ is the electronic effective mass (confinement frequency); the QD lateral length is $l_{0}=\sqrt{\hbar /\left(m \omega_{0}\right)}$. The vertical confinement $V(z)$ is strong enough so that only the state in the first quantum well subband is relevant, and its function is $\varphi_{z}(z)=\sqrt{2 / z_{0}} \sin \left(\pi z / z_{0}\right)$ if a hard wall is assumed, $z_{0}$ being the QD vertical well thickness. In a magnetic field $\mathbf{B}$, the unperturbed Hamiltonian, $H_{0}=\hbar^{2} \mathbf{k}^{2} / 2 m+V(\mathbf{r})+H_{Z}$, has the well-known FockDarwin (FD) solution, where $H_{Z}=g_{0} \mu_{B} \mathbf{B} \cdot \sigma / 2$ is the Zeeman term, $g_{0}$ is the bulk $g$-factor, and $\mathbf{k}$ is the kinetic momentum that includes the magnetic vector potential. We include all SO terms in 2D zincblende QDs, namely Rashba

* present address: Physics Department, University of Ottawa, Ottawa, Ontario K1N6N5, Canada. and Dresselhaus interactions. The former is due to surface inversion asymmetry (SIA) induced by the 2D confinement, while the latter is caused by the bulk inversion asymmetry (BIA) present in zincblende structures. The SIA Hamiltonian is $H_{S I A}=\alpha \sigma \cdot \nabla V(\mathbf{r}, z) \times \mathbf{k}$, while the BIA is $H_{B I A}=$ $\gamma\left[\sigma_{x} k_{x}\left(k_{y}^{2}-k_{z}^{2}\right)+\sigma_{y} k_{y}\left(k_{z}^{2}-k_{x}^{2}\right)+\sigma_{z} k_{z}\left(k_{x}^{2}-k_{y}^{2}\right)\right]$, with coupling constants $\alpha$ and $\gamma$. The $z$-confinement yields the electric field $d V / d z$ in the SIA Hamiltonian as well as the momentum average $\left\langle k_{z}^{2}\right\rangle=\left(\pi / z_{0}\right)^{2}$ in the BIA terms. The full QD Hamiltonian is then $H=H_{0}+H_{S I A}+H_{B I A}$, which is diagonalized in a basis containing 110 FD states. Details about the derivation of $H$ are found elsewhere [5].

We calculate spin relaxation rates between the two lowest Zeeman sublevels caused by piezoelectric and deformation acoustic phonons via Fermi's Golden Rule: $\Gamma_{f i}=$ $2 \pi / \hbar \sum_{j, \mathbf{Q}}\left|\gamma_{f i}(\mathbf{q})\right|^{2}\left|Z\left(q_{z}\right)\right|^{2}\left|M_{j}(\mathbf{Q})\right|^{2}\left(n_{Q}+1\right) \boldsymbol{\delta}\left(\Delta E+\hbar c_{j} Q\right)$, where the sum is over the emitted phonon modes $j$ $(j=L A, T A 1, T A 2)$ with momentum $\mathbf{Q}=\left(\mathbf{q}, q_{z}\right)$. The term $Z\left(q_{z}\right)=\left\langle\varphi_{z}\left|e^{i q_{z} z}\right| \varphi_{z}\right\rangle\left(\gamma_{f i}(\mathbf{q})=\left\langle f\left|e^{i \mathbf{q} \cdot \mathbf{r}}\right| i\right\rangle\right)$ is the form factor perpendicular (parallel) to the $2 \mathrm{D}$-plane (position is $\mathbf{R}=(\mathbf{r}, z)$ ), while $n_{Q}$ is the phonon distribution with energy $\hbar c_{j} Q$; energies $\Delta E=\varepsilon_{f}-\varepsilon_{i}$ and states $|i\rangle,|f\rangle$ are obtained via diagonalization of the total $H$, so that the SO mixing is fully taken into account. The element $M_{j}(\mathbf{Q})=\Lambda_{j}(\mathbf{Q})+i \Xi_{j}(\mathbf{Q})$ includes both piezoelectric $\Lambda_{j}$ and deformation $\Xi_{j}$ potentials; in zincblende structures they become $\Xi_{L A}(\mathbf{Q})=\Xi_{0} A_{L A} \sqrt{Q}$ (only $L A$ is present for $\left.\Xi_{j}\right), \Lambda_{L A}(\mathbf{Q})=3 \Lambda_{0} A_{L A} \sin (2 \theta) q^{2} q_{z} / 2 Q^{7 / 2}$, $\Lambda_{T A 1}(\mathbf{Q})=\Lambda_{0} A_{T A} \cos (2 \theta) q q_{z} / Q^{5 / 2}$, and $\Lambda_{T A 2}(\mathbf{Q})=$ $\Lambda_{0} A_{T A} \sin (2 \theta)\left(2 q_{z}^{2} / q^{2}-1\right) q^{3} / 2 Q^{7 / 2}$ (both $T A 1$ and $T A 2$ modes are compacted as a single $T A$ mode for $\Lambda_{j}$ ), where $A_{j}=\sqrt{\hbar\left(2 N_{0} V c_{j}\right)^{-1}}$ and $\Lambda_{0}=4 \pi e h_{14} / \kappa$. The bulk phonon constants are $\Xi_{0}$ and $e h_{14}, c_{j}$ are the sound velocities $\left(c_{T A} \neq c_{L A}\right), \kappa$ is the dielectric constant, and $N_{0}$ is the electron density. The triple space integral $\left(\left[r, \phi_{r}, z\right]\right)$ allows an analytical form, the same as two $\left(\left[\phi_{q}, q_{z}\right]\right)$ of the momentum integrations, leaving a numerical integral only in $q$. The only $z_{0}$-dependence in this remaining integral in $\Gamma_{f i}$ reads $F_{j}\left(z_{0}\right)=\left(d_{j} z_{0}-\left(d_{j} z_{0}\right)^{3} / \pi^{2}\right)^{-2} \sin ^{2}\left(d_{j} z_{0}\right)$, where $d_{j}=\sqrt{\left(\Delta E / \hbar c_{j}\right)^{2}-q^{2}} / 2 ; q$ runs from 0 to $\Delta E / \hbar c_{j}$, while $F_{j}\left(z_{0}\right)$ is multiplied by polynomials and exponentials in $q$ in the total $\Gamma_{f i}$. No approximation is needed in our derivation 
of $\Gamma_{f i}$, so that the $3 \mathrm{D}$ nature of the phonon is fully taken into account.

Regarding the effective $g$-factor, we consider two possible definitions involving the two lowest Zeeman sublevels for in-plane $\left(B_{\|}\right)$and perpendicular $\left(B_{\perp}\right)$ magnetic field, namely, $g_{\perp, \|}^{E} / g_{0}=\Delta E /\left(g_{0} \mu_{B} B_{\perp, \|}\right)$ or $g_{\perp, \|}^{\sigma_{z}} / g_{0}=\left\langle\Delta \sigma_{z}\right\rangle / 2\left(\left\langle\Delta \sigma_{z}\right\rangle\right.$ is the spin expectation value difference of those levels under $\left.B_{\perp, \|}\right)$. Although the first definition is used operationally in experiments where $\Delta E$ is measured, the latter is intuitively reasonable since $g$ is a quantity intrinsically related to the spin value of those levels. For no SO interaction, both definitions yield $g_{\perp}=g_{\|}=g_{0}$ (no anisotropy).

Figure 1 [9] shows the phonon-induced spin-flip rates as function of in-plane (panel $A$ ) and perpendicular (panel $B$ ) magnetic field, for different values of $E_{0}$, for GaAs QDs [10]. In panel $A$, smaller QDs present smaller spin-flip rates (longer relaxation times). This happens because as $E_{0}$ increases, the orbital levels become more separated and then the SO coupling becomes relatively less important. A strong dependence of the rates with $B_{\|}$and $E_{0}$ is clear. For GaAs QDs under inplane fields, we find that the TA piezoelectric coupling dominates at low fields $\left(<14 \mathrm{~T}\right.$ for $\left.E_{0}=5 \mathrm{meV}\right)$, while at high fields the deformation potential takes over. The experimental spin-flip time at $B_{\|}=10 \mathrm{~T}$ for a $1.1 \mathrm{meV}$ QD is $0.55 \mathrm{~ms}$ [2], while from panel $A$ we find $1.5 \mu \mathrm{s}$. This time increases by one or two orders of magnitude in a larger $z$-well $(\approx 0.05$ $\mathrm{ms}$ at $z_{0}=100 \AA$, not shown), which appears to be a better match for the experimental conditions. A $5 \mu$ s value was found perturbatively $\left(z_{0}=50 \AA, \alpha=0\right)$ [11]. This confirms that the perturbative approach [3] yields the same rates as the exact calculation for GaAs in in-plane fields.
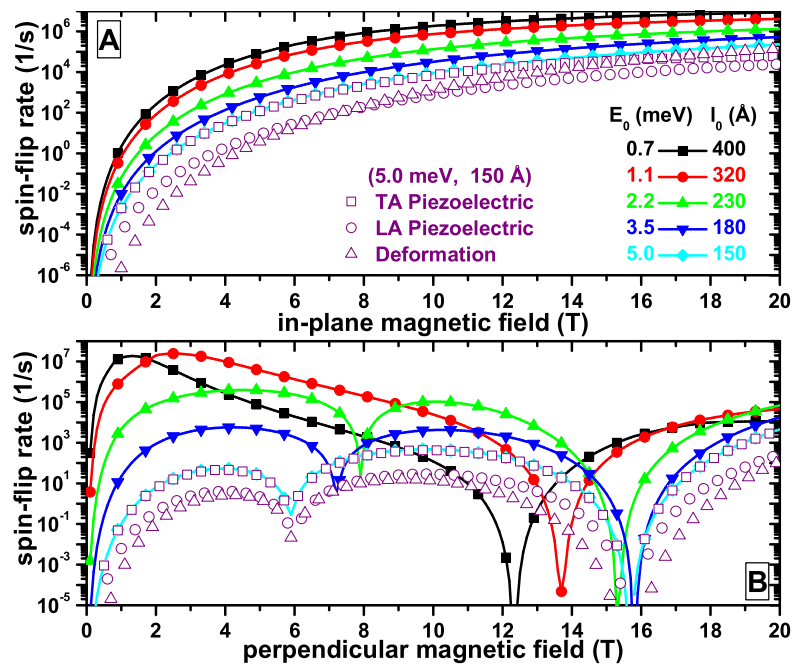

Fig. 1. Zeeman sublevel spin-flip rates for GaAs QDs under in-plane (panel $A$ ) and perpendicular (panel $B$ ) magnetic fields for different QD sizes (solid lines with symbols). Curves with open symbols show contributions from the distinct phonon mechanisms for the $5.0 \mathrm{meV}$ QD; TA piezoelectric is dominating for GaAs. To understand minima at low $B_{\perp}$-field, which are due to a magnetic field-induced cancellation of SO effects, see Fig. 4F.

In panel $B$, the most striking feature under $B_{\perp}$ is the appearance of minima in the rates. The $E_{0}$-dependent minima $\left(12 \leq B_{\perp} \leq 16 \mathrm{~T}\right)$ are due to a vanishing $\Delta E$ (see Fig. $4 F$ ). At such values of $B_{\perp}$, a sublevel crossing produces a sudden spin-flip. To understand the minima at low fields $\left(6 \leq B_{\perp} \leq 8\right.$ T) we have to be mindful of the sine argument in $F_{j}\left(z_{0}\right)$, which may induce a minimum in the rate at a particular value of the field, due to the interplay of the energy scales in the problem. We find that the three low-field minima occur at $B_{\perp}$-values where a magnetic field-induced cancellation of the SO influence is produced on the respective $\Delta E$ values (see Fig. $4 F$ ): below (above) such $E_{0}$-dependent values of $B_{\perp}-$ where $\Delta E$ recovers its pure Zeeman value of $g_{0} \mu_{B} B$ - the SO coupling increases (decreases) the sublevel splitting as compared to the QD without SO. Notice that the two smallest confinements do not show the low-field minima. This is due to the lowest sublevels acquiring the same spin (between 3 and $7 \mathrm{~T}$ at 0.7 $\mathrm{meV}$, not shown), so that the rate decreases monotonically at that field-range. Like in the $B_{\|}$case, the dominating phonon mechanism is the TA piezoelectric coupling, but now at any field. Our results agree with available calculations [4] at small fields $\left(B_{\perp} \leq 1 \mathrm{~T}\right)$, so that we can confirm that the perturbative approach is not adequate when dealing with SO effects in QDs in a perpendicular field (even in GaAs) if the vertical confinement is strong enough [12]. Notice that rates at small fields $(<2 \mathrm{~T})$ have the same behavior under $B_{\|}$and $B_{\perp}$ : the smaller $E_{0}$ the larger the rate; however, they are much larger under $B_{\perp}$.
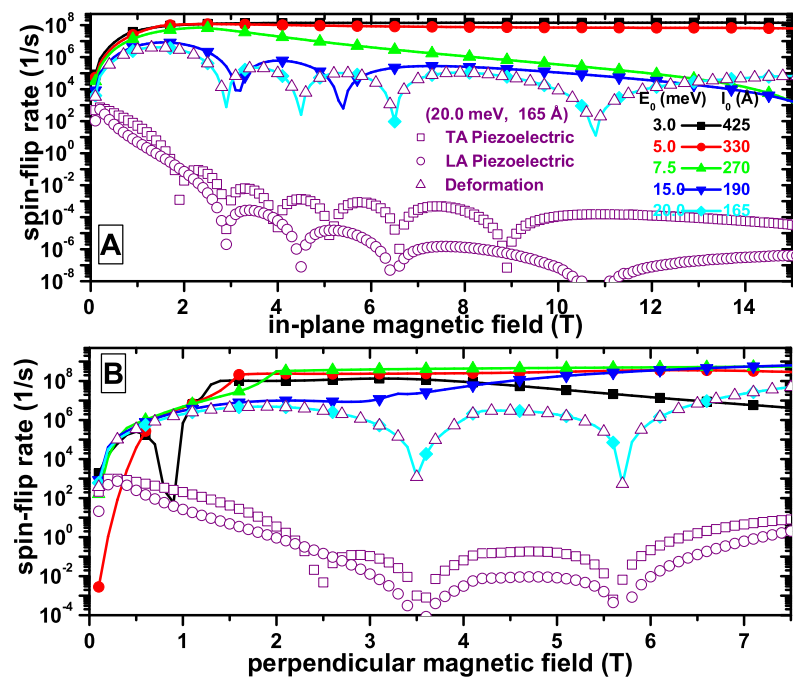

Fig. 2. Same as Fig. 1, but for InSb QDs. The confinements are such that the same QD size-range used for GaAs is covered. The 20.0 $\mathrm{meV}$ QD is chosen for the study of distinct phonon mechanisms, where the LA deformation potential is shown to dominate. Both LA modes have the same number of minima, which occur at the same field-values. The number of minima increases with $E_{0}$. Minimum of the $3.0 \mathrm{meV}$ QD at $B_{\perp}=1 \mathrm{~T}$ indicates a sublevel crossing (see Fig. $3 \mathrm{~F})$.

Figure 2 [9] shows results for InSb QDs [13] in in-plane (panel $A$ ) and perpendicular (panel $B$ ) magnetic fields. In panel $A$, in contrast to GaAs, minima in the rates are also visible under $B_{\|}$for the highest $E_{0}$ values. At small fields $(<2$ $\mathrm{T})$, a monotonic rate drop is again observed as $E_{0}$ increases. 
As shown for the $20 \mathrm{meV} \mathrm{QD}$, the LA deformation potential is the dominant phonon mechanism for InSb at any field-value (and $E_{0}$ ). It is worth mentioning that, contrary to GaAs, where a unique low $B_{\perp}$-field minimum is present for a given $E_{0}$, the number of rate minima in InSb increases with $E_{0}$. Notice that both LA modes have the same number of minima - occurring at the same $B_{\|}$-values -, while a larger number of minima in the TA mode occurs at different field-values; this happens because $c_{L A}>c_{T A}$, so that the sine argument in $F_{j}\left(z_{0}\right)$ is smaller in the LA mode. Notice that the oscillatory rate is well defined until the Zeeman sublevels acquire the same spin; e.g., for a $15 \mathrm{meV}$ QD, that occurs around $14 \mathrm{~T}$.

In panel $B$, all spin-flip rates are well defined only for $B_{\perp}<$ $8 \mathrm{~T}$. Such a field is even smaller (around $2 \mathrm{~T}$ ) for small $E_{0}$, as it occurs for the $3.0 \mathrm{meV} \mathrm{QD}$; the rate minimum around $1 \mathrm{~T}$ for this $E_{0}$ indicates a spin-flip, accompanied by the vanishing of $\Delta E$ (see Fig. $3 F$ ). Larger values of $E_{0}$ do not present spin-flip. The relaxation rates in panel $B$ do not show the monotonic behavior seen at small fields for the in-plane field direction. Like in the $B_{\|}$case, the LA deformation potential dominates at any field-value. We emphasize that the perturbative approach finds no use in InSb QDs because of the inherent higher SO coupling.

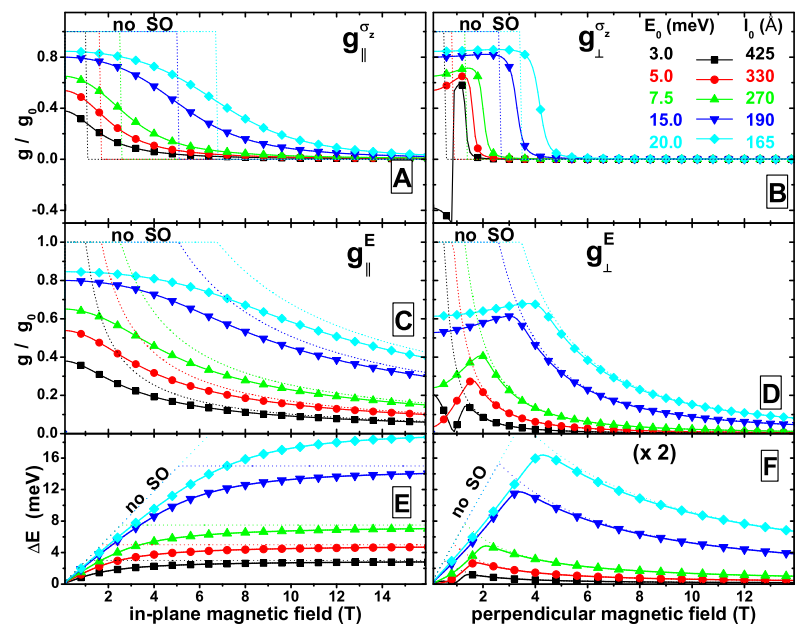

Fig. 3. Electron $g$-factor of InSb QDs with different $E_{0}$ values. Left (right) panels refer to in-plane (perpendicular) field. Panels $A$ and $C$ $\left(B\right.$ and $D$ ) show $g_{\|}\left(g_{\perp}\right)$, while panels $E$ and $F$ show sublevel splitting $\Delta E$ for the two lowest QD states. Panels $A$ and $B(C$ and $D)$ show $g$ as obtained from the $\left\langle\Delta \sigma_{z}\right\rangle(\Delta E)$ calculation. Dotted lines in all panels refer to corresponding QDs without SO coupling. $\Delta E$ in panel $F$ is multiplied by 2 .

Figures 3 [14] (InSb, [13]) and 4 [14] (GaAs, [10]) present the effective $g$-factor for different $l_{0}$ sizes, with left (right) panel for in-plane (perpendicular) fields, while dotted lines show results without SO coupling. Panels $A$ and $B(C$ and $D)$ use the definition of $g$ in terms of $\left\langle\Delta \sigma_{z}\right\rangle(\Delta E)$. The respective sublevel energy splitting is shown in panels $E$ and $F$. Notice that for InSb both definitions yield basically the same low $B_{\|}$field results. The drop in $g_{\|}$is faster in the $g^{\sigma_{z}}$ curves because the two lowest states acquire the same spin at higher fields. In the low $B_{\perp}$-field results, however, the two $g$-factor defin- itions yield different values mainly at weaker confinements, although the drop in $g_{\perp}$ is also faster in the $g^{\sigma_{z}}$ curves. Observe that for the smallest $E_{0}$ (largest $\mathrm{SO}$ effect) of $3.0 \mathrm{meV}$, a sign change is seen in $g_{\perp}^{\sigma_{z}}$ around $1 \mathrm{~T}$, which relates to an unusual crossing involving the ground and first excited states. In both field-directions, smaller $E_{0}$ yields smaller $g$-factor, which shows that SO coupling provides a channel to manipulate $g$ in QDs under magnetic fields. Notice the clear anisotropy in $g^{E}$ (panels $C$ and $D$ ): the same QD confinement shows $g_{\perp}^{E}<g_{\|}^{E}$, since the mixing with higher orbitals is stronger for $B_{\perp}$. If the $g^{\sigma_{z}}$ definition is considered (panels $A$ and $B$ ), such anisotropy is not as remarkable at low fields.

Figure 4 shows that for GaAs both definitions in panels $A$ and $C$ give essentially the same $g_{\|}$-values. Results are totally different in a perpendicular field. At $1.1 \mathrm{meV}$, it can be seen (panel $B$ ) that $g_{\perp}^{\sigma_{z}}$ has inverted sign at low fields, becomes zero for $B_{\perp}$ between 4 and $8 \mathrm{~T}$, then acquires inverted sign again, and suddenly flips back to its 'normal' behavior at 13.7 $\mathrm{T}$; under higher fields, $g_{\perp}^{\sigma_{z}}$ goes to zero since the two lowest level spins are aligned. Panel $B$ also shows that larger $E_{0^{-}}$ values (smaller SO coupling) cancels the field range where $g_{\perp}^{\sigma_{z}}$ is zero. Notice that the weaker the confinement the smaller the field where the sign change occurs.

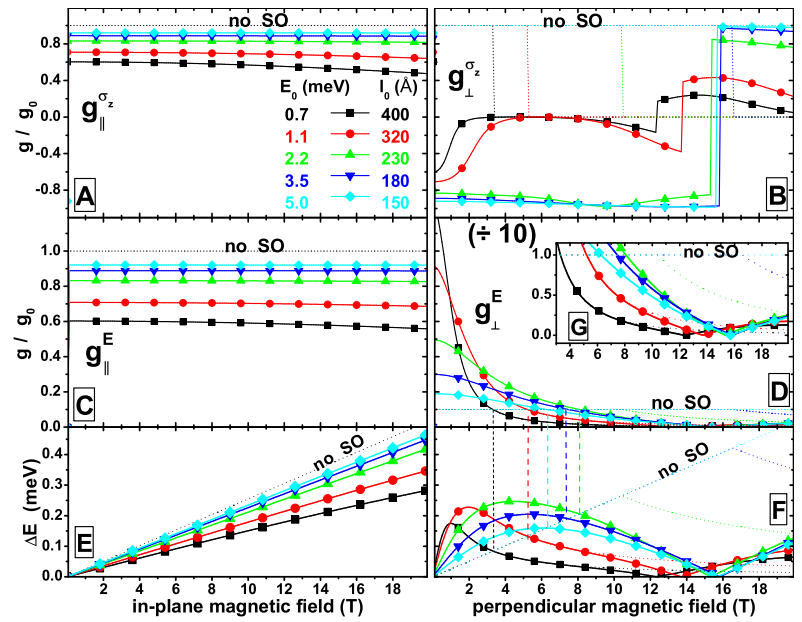

Fig. 4. Same as Fig. 3, but for GaAs QDs. $g_{\perp}^{\sigma_{z}}$ has inverted sign at low fields, indicating that SO coupling is stronger than the pure Zeeman splitting. Dashed lines connecting panels $D$ and $F$ show fields for which $g_{\perp}^{E} / g_{0}=1$, where the SO effect is effectively cancelled by the magnetic field. $g$ in $D$ is divided by 10 .

One finds totally different results for $g_{\perp}^{E}$ (panel $D$ ), which may even assume values 12 times larger than $g_{0}$ for the smallest $E_{0}$ at low fields; still at low fields, larger $E_{0}$ tend to reduce $g_{\perp}^{E}$ towards $g_{0}$. At high fields (inset $G$ ), $g_{\perp}^{E}$ goes to zero when the level crossing involving the ground state occurs. For every $E_{0}$ there is a magnetic field - indicated by the dashed lines connecting panels $D$ and $F$ - where $g_{\perp}^{E}$ goes from higher to smaller values than $g_{0}$ (see discussion for the low-field minima in Fig. 1B). Such result emphasizes the intricate competition between external magnetic field and intrinsic SO coupling in QDs [6]. In GaAs QDs, the anisotropic nature of the $g$-factor is much more pronounced, despite the small values 
of the SO constants. For an experimental comparison [8], at GaAs QD with $E_{0}=1.1 \mathrm{meV}$ and $B_{\|}=10 \mathrm{~T}, \Delta E \simeq 200 \mu \mathrm{eV}$ was reported, while from Fig. $4 \mathrm{E}$ one finds $\Delta E \simeq 180 \mu \mathrm{eV}$; in a linear fit, $|g|=0.29 \pm 0.01$ was found and from Fig. $4 A(4 C)$ one has $\left|g_{\|}^{\sigma_{z}}\right|=0.30\left(\left|g_{\|}^{E}\right|=0.31\right)$.

Even though both QD materials show minima in the spinflip rates - with $B_{\perp}$ for GaAs and with $B_{\|}$and $B_{\perp}$ for $\mathrm{InSb}-$ their origin is slightly different. Minima come from the nature of the $z$-confinement, and the field where they occur depend on $l_{0}$. The SO coupling mixes spins and alters splitting of sublevels in distinct ways according to field-direction and QD material, so that spin relaxation can be induced by piezo- electric (wide-gap) and deformation (narrow-gap) phonons. The external field opens channels (at the rate minima) where long spin relaxation times $(\simeq 1 \mathrm{~s})$ may be reached, so that the spin coherence required for quantum computing could be improved. The SO coupling is able to tune the anisotropic electron $g$-factor in QDs and even change its sign. There is a complex interplay between SO and magnetic energies, which creates distinct phases in the GaAs QD spectra under $B_{\perp}$, where the SO interaction increases or decreases the Zeeman sublevel splitting. These features would not have been accessed if a perturbative approach had been used, especially for QDs with large (small) lateral (vertical) size.
[1] D. Loss et al., Phys. Rev. A 57, 120 (1998).

[2] J. M. Elzerman et al., Nature 430, 431 (2004).

[3] A. V. Khaestkii et al., Phys. Rev. B 64, 125316 (2001); L. M. Woods et al., Phys. Rev. B 66, 161318(R) (2002).

[4] J. L. Cheng et al., Phys. Rev. B 69, 115318 (2004).

[5] C. F. Destefani et al., Phys. Rev. B 69, 125302 (2004); C. F. Destefani et al., Phys. Rev. B 70, 205315 (2004).

[6] M. Valín-Rodríguez, Phys. Rev. B 70, 033306 (2004).

[7] G. Medeiros-Ribeiro et al., Appl. Phys. Lett. 80, 4229 (2002).

[8] R. Hanson et al., Phys. Rev. Lett. 91, 196802 (2003).

[9] C. F. Destefani et al., Phys. Rev. B, 72, 115326 (2005).

[10] $m / \mathrm{m}_{0}=0.067, g_{0}=-0.44, \kappa=12.4, \alpha=4.4 \AA^{2}, \gamma=26 \mathrm{eV} \AA^{3}$,
$\Xi_{0}=7 \mathrm{eV}, e h_{14}=0.140 \mathrm{eV} / \AA, N_{0}=5.32 \times 10^{-27} \mathrm{Kg} / \AA^{3}, c_{L A}=$ $4.73 \times 10^{13} \AA / \mathrm{s}, c_{T A}=3.35 \times 10^{13} \AA / \mathrm{s}, z_{0}=40 \AA, d V / d z=$ $-0.5 \mathrm{meV} / \AA \AA$.

[11] V. N. Golovach et al., Phys. Rev. Lett. 93, 016601 (2004).

[12] If a Gaussian well is considered, one has $\left\langle k_{z}^{2}\right\rangle=1 / z_{0}^{2}$, a BIA term a factor of 10 smaller than used in this work.

[13] $m / \mathrm{m}_{0}=0.014, g_{0}=-51, \kappa=16.5, \alpha=500 \AA^{2}, \gamma=160 \mathrm{eV} \AA^{3}$, $\Xi_{0}=7 \mathrm{eV}, e h_{14}=0.061 \mathrm{eV} / \AA, N_{0}=5.77 \times 10^{-27} \mathrm{Kg} / \AA^{3}, c_{L A}=$ $3.40 \times 10^{13} \AA / \mathrm{s}, c_{T A}=2.29 \times 10^{13} \AA / \mathrm{s}, z_{0}=40 \AA, d V / d z=$ $-0.5 \mathrm{meV} / \AA$.

[14] C. F. Destefani et al., Phys. Rev. B 71, 161303(R), 2005. 